\title{
Efficacy of a Sclerostin Antibody Compared to a Low Dose of PTH on Metaphyseal Bone Healing
}

Fredrik Agholme, Brandon Macias, Matt Hamang, Jonathan Lucchesi, Mary D. Adrian, Stuart Kuhstoss, Anita Harvey, Masahiko Sato and Per Aspenberg

\section{Linköping University Post Print}

\section{Tweet}

N.B.: When citing this work, cite the original article.

Original Publication:

Fredrik Agholme, Brandon Macias, Matt Hamang, Jonathan Lucchesi, Mary D. Adrian, Stuart Kuhstoss, Anita Harvey, Masahiko Sato and Per Aspenberg, Efficacy of a Sclerostin Antibody Compared to a Low Dose of PTH on Metaphyseal Bone Healing, 2014, Journal of Orthopaedic Research, (32), 3, 471-476.

http://dx.doi.org/10.1002/jor.22525

Copyright: Wiley http://eu.wiley.com/WileyCDA/

Postprint available at: Linköping University Electronic Press http://urn.kb.se/resolve?urn=urn:nbn:se:liu:diva-103861 


\section{Efficacy of a Sclerostin Antibody Compared to a Low Dose of PTH on Metaphyseal Bone Healing}

${ }^{1}$ Agholme, Fredrik

${ }^{1}$ Macias, Brandon

${ }^{2}$ Hamang, Matt

${ }^{2}$ Lucchesi, Jonathan

${ }^{2}$ Adrian, Mary D

${ }^{2}$ Kuhstoss, Stuart

${ }^{2}$ Harvey, Anita

${ }^{2}$ Sato, Masahiko

${ }^{1}$ Aspenberg, Per

\section{Affiliation:}

${ }^{1}$ Orthopaedics, Department of Clinical and Experimental Medicine, Linköping University, Linköping, Sweden

${ }^{2}$ Lilly Research Laboratories, Indianapolis, IN, USA

\section{Corresponding Author:}

Per Aspenberg

Ortopedi

Universitetssjukhuset

SE-581 85 Linköping

Sweden

per.aspenberg@liu.se

+4610-1034166

Running title:

Sclerostin antibody versus PTH 


\begin{abstract}
We compared the effect of a sclerostin antibody to that of a clinically relevant dose of PTH in a rat model for metaphyseal bone healing. Screws of steel or PMMA were inserted bilaterally into the proximal tibia of young male rats. During 4 weeks the animals then received injections of PBS (control), sclerostin antibody (25 mg/kg, twice weekly) or PTH (5 $\mu \mathrm{g} / \mathrm{kg}$, daily). The healing response around the screws was then assessed by mechanical testing and $\mu \mathrm{CT}$. To distinguish between effects on healing and general effects on the skeleton, other untraumatized bone sites and serum biomarkers were also assessed. After 4 weeks of treatment, PTH yielded a 50\% increase in screw pull-out force compared to control (p $=0.03$, while the antibody had no significant effect. In contrast, the antibody increased femoral cortical and vertebral strength where PTH had no significant effect. $\mu \mathrm{CT}$ showed only slight changes that were statistically significant for the antibody mainly at cortical sites. The results suggest that a relatively low dose of PTH stimulates metaphyseal repair (screw fixation) specifically, whereas the sclerostin antibody has wide-spread effects, mainly on cortical bone, with less influence on metaphyseal healing.
\end{abstract}

\title{
Keywords
}

Implant fixation, Bone healing, mechanical testing, bone formation, Wnt-signaling 


\section{Introduction}

Fractures have a high morbidity in the elderly, due to the long healing time of fractures and the immobility and suffering that often occurs as a consequence of the injury. Therapies that increase bone formation and reduce healing time would be beneficial for these patients. Two investigational agents that have been suggested as potential augmenters of fracture healing include sclerostin antibodies and parathyroid hormone (PTH).

The majority of osteoporotic fractures in humans occur at corticocancellous sites such as in vertebra or in metaphyseal regions. Because fractures in cancellous bone occur in an environment with abundant mesenchymal stem cells, the healing process faces fewer challenges than in shaft fractures, and might comprise only parts of the more complex array of events that are involved in these. Pharmacological stimulation of fracture healing in cancellous bone should therefore probably aim at accelerating the local bone formation that was initiated by the trauma. Animal models of fracture healing in cancellous bone are rare Ref Histing et al. Bone 2011. One way to estimate cancellous healing in mechanical terms is to implant a screw in cancellous bone and measure the pull-out force. The trauma of drilling and inserting the screw leads to a fracture healing response that initiates new bone formation in the vicinity of the screw REF Wermelin Acta och Wermelin Bone. As this leads to increasing strength of the bony threads holding the screw, the pull-out force increases over time, and reflects the efficacy of the healing response.

Sclerostin is a secreted glycoprotein and product of the SOST gene. Sclerostin antagonizes Wnt signaling [1] and appears to be a potent negative regulator of bone formation [2, 3]. Wnt signaling plays an important role in bone tissue and precise regulation is needed for a proper bone healing response [4]. In bone, only osteocytes express sclerostin, since it is exclusively expressed by terminally differentiated cells in mineralized tissues [5]. Loss of function mutations in sclerostin leads to excessive bone formation [2, 6, 7] and causes Sclerosteosis and Van Buchems disease [2, 8-10]. By inactivating 
sclerostin with an antibody, fracture healing can be improved in rats [11] and nonhuman primates [12]. Furthermore, clinical trials of sclerostin antibodies in bone healing have started but results have not yet been reported.

Intermittent dosing of PTH is an osteoporosis treatment that can stimulate fracture healing [13-16] as well as implant fixation $[17,18]$ in animals. However, in subsequent clinical trials only a modest effect on human fracture healing was detected with PTH $[19,20]$. The difference between results from animal experiments and human trials could be due to the fact that PTH, in humans, can only be given at doses lower than those normally used in animal experiments.

In this study we compared the effects of a sclerostin antibody (Scl-ab) to that of a more clinically relevant dose of PTH in a rat model of metaphyseal fracture healing, based on screw pull-out resistance. We hypothesized that Scl-ab would be more efficacious than PTH in this model since Scl-ab can be given at higher doses.

\section{Materials \& Methods}

Animals and surgery: A total of 30 male, 3 month old Sprague Dawley rats with a mean weight of $360 \pm 30$ grams were used. Under general anesthesia, a hole was drilled in the anterior-medial surface of the proximal tibia on both hind legs. In the right leg, a stainless steel screw (thread: M1.7, length of threaded part $2.8 \mathrm{~mm}$ ) was inserted in the metaphyseal bone close to the physis. In the left leg, a poly methyl methacrylate (PMMA) screw of similar dimensions was inserted at the same site. After surgery, the animals were randomly allocated by lottery into three treatment groups $(\mathrm{N}=10)$ and subcutaneously administered either PBS vehicle (control), sclerostin antibody (Scl-ab) $25 \mathrm{mg} / \mathrm{kg}$ given twice a week, or Teriparatide (PTH) $5 \mu \mathrm{g} / \mathrm{kg}$ given daily. All injections were given subcutaneously and started the day after surgery. 4 weeks after surgery the animals were euthanized using an intracardial injection of pentobarbital. Subsequently the tibiae, femora and lumbar 1-5 vertebrae, as well as serum were 
harvested. Analysis of tibiae was conducted by the Aspenberg lab in Linköping while femora and vertebrae were further analyzed at the Sato lab at Lilly. All animal experiments were approved by the regional board for the welfare of experimental animals. All treatment agents were supplied by Lilly.

Mechanical testing: Steel screws were tested for pull-out strength using a computerized materials testing machine at a cross head speed of $0.1 \mathrm{~mm} / \mathrm{s}$ - The peak pull-out force was considered the main variable. During analyses the operator was blinded for treatment.

Femora and vertebra were excised at necropsy, cleaned of soft tissue, wrapped in saline soaked gauze and stored at $-20 \mathrm{oC}$ or below. Femora were loaded to failure in 3-point-bending in a $37 \mathrm{oC}$ saline bath after being submerged for 2 min to allow for equilibration of temperature. Load-displacement curves were recorded at a crosshead speed of $0.17 \mathrm{~mm} / \mathrm{sec}$ using an MTS model 1/S materials testing machine with TestWorks 4 software (MTS Corp., Minneapolis, MN). Midshaft and proximal femur specimens were prepared separately, before testing. Ultimate load for the femoral neck was measured by mounting the proximal half of the femur vertically in a chuck at room temperature and applying a downward for on the femoral head until failure [21]. The ultimate load was measured as the maximum force sustained by the femoral neck and was considered to be an estimate of femoral neck strength. All tests were conducted using the materials testing machine and analyzed using TestWorks 4 software (MTS Corp.). Mechanical properties of L-5 vertebrae were analyzed after the posterior processes were removed, and the ends of the centrum were made parallel using a diamond wafering saw (Buehler Isomet, Evanston, IL). Vertebral specimens were loaded to failure in compression, using the materials testing device and analyzed using TestWorks 4 software (MTS Corp.). Specimens were tested in a saline solution at $37 \mathrm{oC}$, after equilibration.

$\boldsymbol{\mu C T}$ imaging: Tibiae containing PMMA screws were subjected to $\mu \mathrm{CT}$ scanning with an isotropic voxel size of $10 \mu \mathrm{m}$ (Skyscan 1174, v. 2, Skyscan, Aarteselar Belgium) using aluminum filter of 0.5 
$\mathrm{mm}$, and 5 repeated scans. The images were reconstructed using NRecon, by correcting for ring artifacts and beam hardening. A cylindrical $2 \mathrm{~mm}$ wide region of interest was applied around the screw and extended to the length of the screw (approximately $3 \mathrm{~mm}$ ). This region of interest was then divided into two parts: cortical region and marrow region (Figure 1) $[11,22]$. Bone volume / tissue volume (BV/TV) was considered the primary variable. All analyses were performed in a blinded manner. In addition, the distal femur metaphysis and midshaft were analyzed post-necropsy using an Aloka LaTheta LTC-100 uCT (Aloka, Tokyo, Japan) to measure Bone mineral density (BMD) and bone mineral content (BMC). Bones were mounted on modeling clay for positioning and regions of the femora were analyzed at $2 \mathrm{~mm}$ from the end of the growth plate for distal metaphysis and at the midshaft, respectively. Only the left leg was scanned.

Serum bone markers: Serum osteocalcin was measured by EIA using a kit from Biomedical Technologies, Inc. (Stoughton, MA). Serum C-terminal telopeptides of type I collagen (CTX1) were analyzed using a commercially available kit (RatLaps; Immunodiagnostic Systems Ltd., Fountain Hills, AZ) following the manufacturer's protocol. Similarly, procollagen type I N-terminal propeptide (PINP) levels were determined using an ELISA kit (Immunodiagnostic Systems Inc., Fountain Hills, AZ). Antibody levels were also measured with an antigen-down format ELISA.

Statistics: Data were tested for normal distribution and equivalence of variances. ANOVA followed by t-test, with Bonferroni correction for multiple testing, was used for statistical evaluation. Data with P $<0.05$ was considered significant, $95 \%$ confidence interval $(\mathrm{CI})$ for \%-change of treatment mean versus control or other treatment mean are provided. 


\section{Results}

\section{Metaphyseal healing (Screw fixation)}

Bone healing efficacy was evaluated at study termination post-necropsy. After 4 weeks of treatment, PTH yielded a $48 \%$ (CI 4 to 91) increase in screw pull-out force compared to control (Figure 2). Scl-ab increased the pull-out force by $27 \%$ (CI -16 to 71 ). A direct comparison of PTH and the sclerostin antibodies showed a statistically insignificant difference in pull-out force by $16 \%$ (CI -18 to 50 ). $\mu \mathrm{CT}$ analyses showed that neither treatment significantly increased the BV/TV in the entire region adjacent to the PMMA screws. However, in the cortical region, Scl-ab caused a significant increase in BV/TV $(\mathrm{p}=$ 0.04): there was no significant increase in cancellous bone density in the marrow (Figure 3). Images of the median specimen in each group are shown (Figure 4).

\section{Other sites}

Other bone sites and sera were evaluated to clarify systemic pharmacological efficacy of treatments. PTH had no significant effect on load to failure at 3-point bending of the femoral shaft or proximal femur. In contrast Scl-ab treatment caused a $41 \%$ (CI 11 to 71) increase in peak load of the proximal femora, compared to controls. Also in the femoral midshaft, a 17\% (CI 6 to 28) increase in peak load was detected following Scl-ab treatment (Figure 2). Similarly, compression testing showed a $47 \%$ (CI 8 to 85) stronger and tougher vertebra following Scl-ab treatment) (Figure 2). At all these sites, a direct comparison showed significantly stronger effects of the sclerostin antibodies compared to PTH $(\mathrm{p}<0.05$, for all).

In the distal femur metaphysis, both PTH and Scl-ab increased BMD and BMC significantly, relative to controls (Figure 5). The effect of PTH was 15\% (CI 7 to 23) versus Scl-ab 9\% (CI 1 to 17) but there was no statistically significant difference between PTH and Scl-ab. In the femoral midshaft there was no 
effect of either treatment on BMD, but Scl-ab treatment showed a 10\% (CI 4 to 17) increase in BMC (Figure 5).

Serum osteocalcin levels were elevated with PTH $(\mathrm{p}=0.05)$ but not with Scl-ab relative to controls (Figure 6). There were no differences between groups for PINP or CTX-1 (Figure 5). In addition, serum analysis confirmed a robust systemic exposure with the sclerostin antibody at study termination (data not shown). 


\section{Discussion}

The response to the trauma of inserting a screw in cancellous bone appears similar to the healing response after a metaphyseal fracture, although the geometry of the injury is different. The repair process leads to the formation of bony threads, increasing the hold of the screw dramatically after insertion [23]. By measuring pull-out force for screws in cancellous bone we can get an estimate for the strength of this new bone, i.e. metaphyseal bone formation in response to trabecular disruption. Although theoretically this would correspond to fracture healing, it can not be excluded that when cancellous bone is crushed, such as in most fractures, that other processes may also be involved. We could not confirm our hypothesis of superior sclerostin antibody efficacy in cancellous bone healing relative to PTH. In contrast, we found a surprising efficacy of a relatively low dose of PTH to enhance metaphyseal bone healing. Serum osteocalcin and cancellous bone data further confirmed systemic efficacy of a low dose of PTH, although for PINP and CTX-I we saw no significant changes indicating that systemic effects on bone turnover were small. Previous studies in this model have shown similar positive effects of larger doses of PTH that may not be clinically relevant [18]. The currently used dose of $5 \mu \mathrm{g} / \mathrm{kg} / \mathrm{day}$ of PTH has been shown to yield a systemic exposure in rats of about 1.6 to 3 times the clinical dose in humans, as estimated from the area under the curve of serum concentration over time [24]. An intact Wnt signaling is required for PTH to function properly [25, 26] suggesting an interaction between these two pathways. Moreover, SOST is a target of PTH, suggesting that some of the effect of PTH might be mediated via Wnt signaling [27]. However, our results suggest that even though similar pathways may be involved, PTH and SOST antibodies affect the anabolic response in bone differently.

Sclerostin antibodies have been shown to improve bone formation in several models and also to improve implant fixation and bone healing $[11,28]$. The limited effect on implant fixation in this experiment contrasts to an earlier study in which we saw a positive effect on bone healing in a similar model [11]. However, the magnitude of the effect on bone healing of the antibody in the two 
experiments is not dramatically different: increase in pull-out force by 52\% (CI 33 to 71 ) in the earlier study versus $27 \%$ (CI -16 to 71 ) in this study. Although the antibodies used in the two studies are derived from different sources, this is unlikely to explain the differences, as we saw clear effects of the antibody used in this study on cortical bone. However, it is possible that differences in epitopes between the two antibodies lead to a different outcome due to the complex interaction between SOST and its receptors Lrp4/5/6 [10, 29]. However, the most striking finding in this study is not the weaker effect of the antibody, but the robust effect of low dose PTH on healing.

Our data appear to support the notion that clinically relevant doses of PTH have a beneficial effect on cancellous bone healing, while sclerostin suppression seems to have a larger systemic effect, mainly affecting cortical bone. Previous studies of higher doses of PTH on screw fixation in this model showed striking effects, but also then, there was an absence of a notable increase in cancellous bone density in either the distal femoral diaphysis or vertebral bodies, indicating that the effect of PTH on bone healing are more dramatic than during ordinary background remodelling [17, 18]. The effects on pull-out force were significant, but changes in morphological parameters were not. Although this could reflect a discrepancy between effects on bone quality versus the amount of bone, we believe it more likely that it is related to a difference in sensitivity between mechanical versus morphometric methods.

There is a concern that inhibition of sclerostin in patients would lead to complications similar to sclerosteosis. However, humans that are heterozygote carriers of these mutations do not present with a severe phenotype but instead have a higher than normal bone mass [30, 31]. For these reasons, treating adults for short periods of time with sclerostin antibodies should not pose a risk greater than those for heterozygote individuals with SOST mutations. 
In conclusion, a low dose of PTH enhanced metaphyseal repair, whereas the sclerostin antibody had mainly cortical bone effects with less influence on metaphyseal healing; therefore new clinical studies of PTH treatment for accelerating corticocancellous fracture repair appear warranted. 
Figures
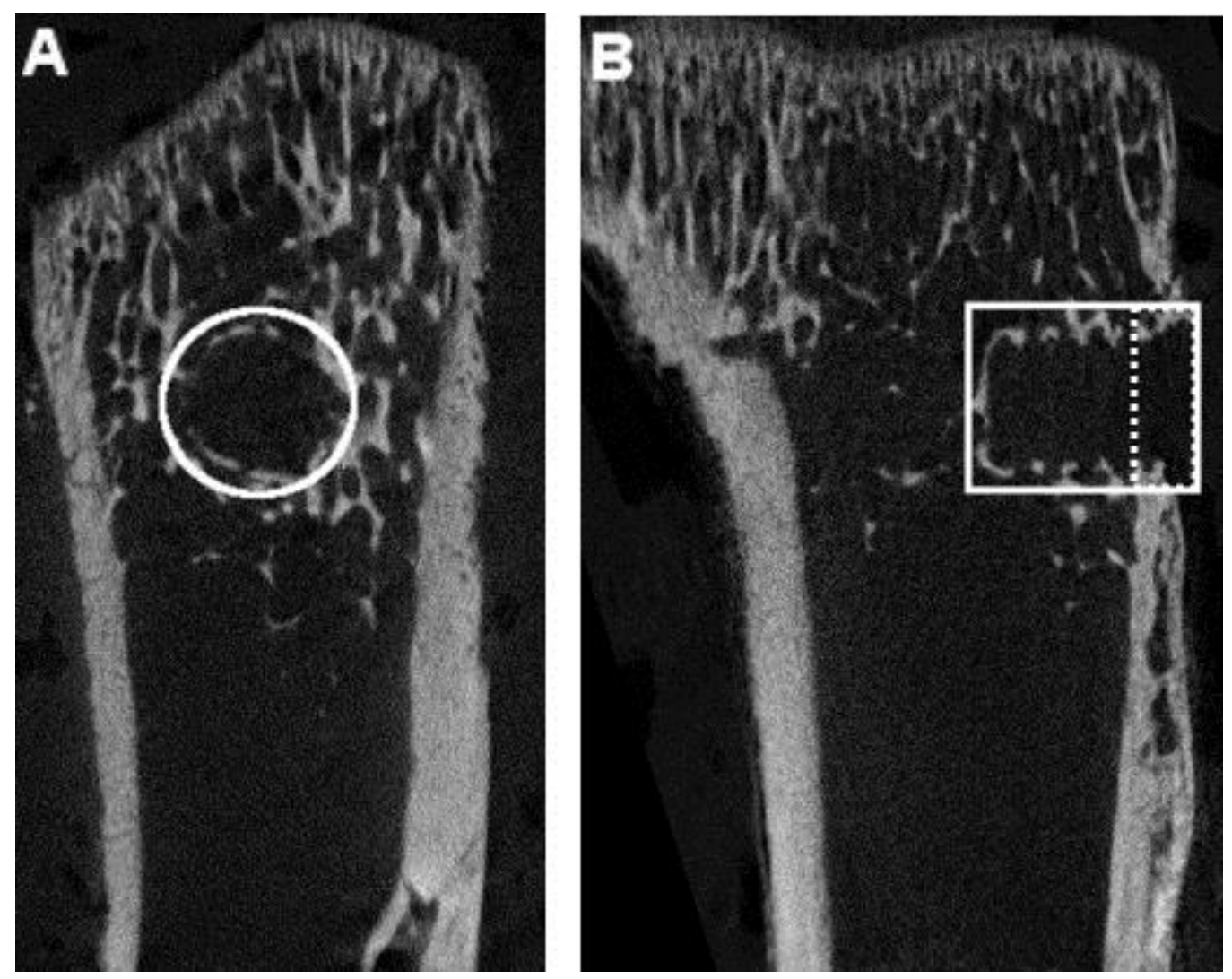

Figure 1: Tibial cross-sections illustrating ROI (white outline) for $\mu \mathrm{CT}$ measurements. The PMMA screw is not visible. A a cylindrical $2 \mathrm{~mm}$ wide section centered on the screw is selected. B the cylinder extends the length of the screw. The ROI is dived into a cortical region (right of dashed line) and marrow region. 

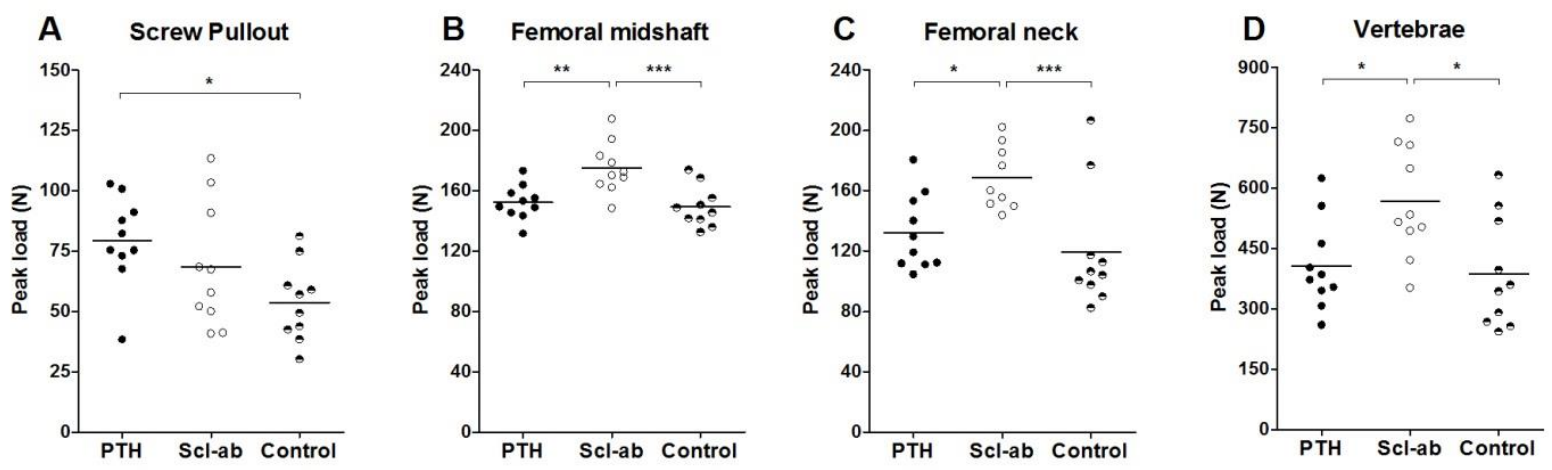

Figure 2: Mechanical measurements after 4 weeks of treatment. A) PTH increased the pull-out strength of the steel screws in the metaphyseal bone. B) Scl-ab increased the three-point bending strength of the femoral midshaft compared both with controls and PTH. C) Also in the femoral neck Scl$\mathrm{ab}$ increased the strength of the bone compared to controls and PTH D) Scl-ab also increased the compression strength of the lumbar-5 vertebrae both compared with controls and PTH. *, p < $0.05 ; * *, p$ $<0.01 ; * * *, \mathrm{p}<0.001$ 

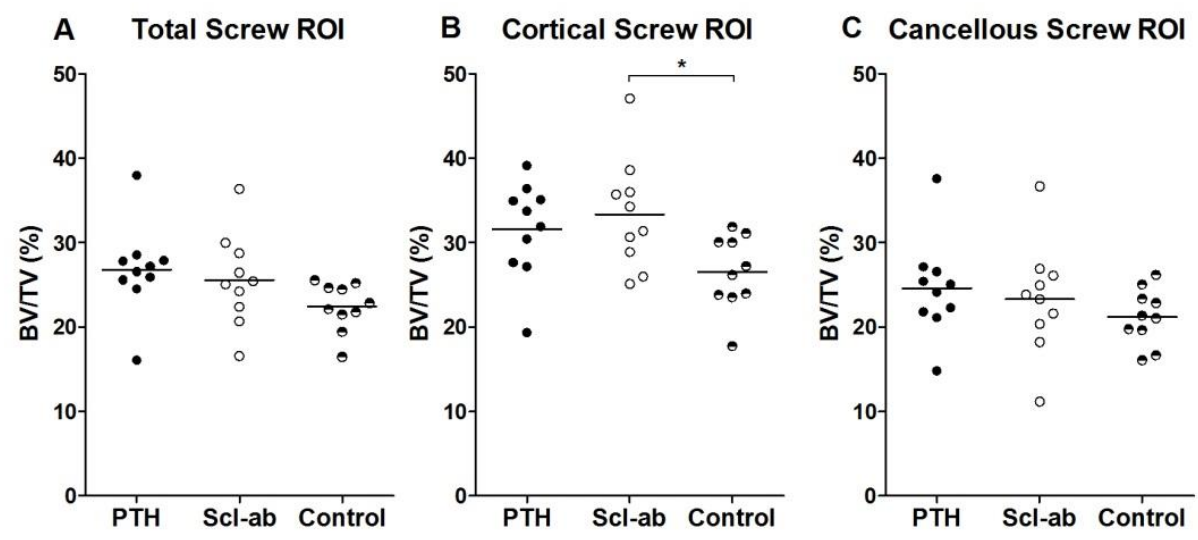

Figure 3: $\mu \mathrm{CT}$ measurements of the screw region of interest (ROI) in the proximal tibia at 4 weeks.

A) Neither PTH nor Scl-ab treatment caused a significant increase in bone volume fraction (BV/TV) in the total screw surrounding. B) However, in the cortical ROI Scl-ab had a significant effect on BV/TV C) In the cancellous ROI there was no significant effect of either treatment. *, p < 0.05 ; 

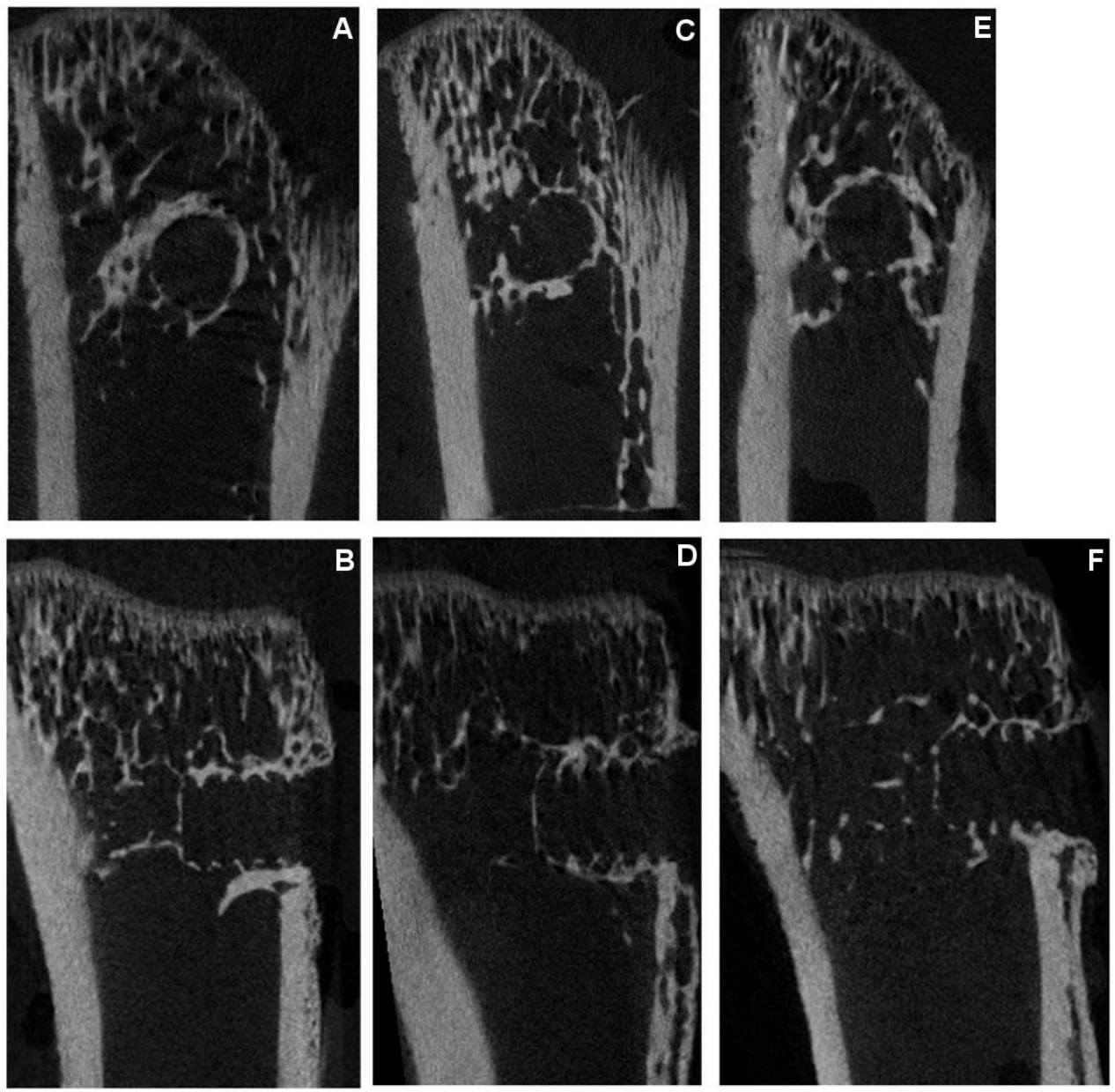

Figure 4: Representative $\mu$ CT tibial cross-sections. A, B PTH. C,D Control. E, F Scl-ab. 

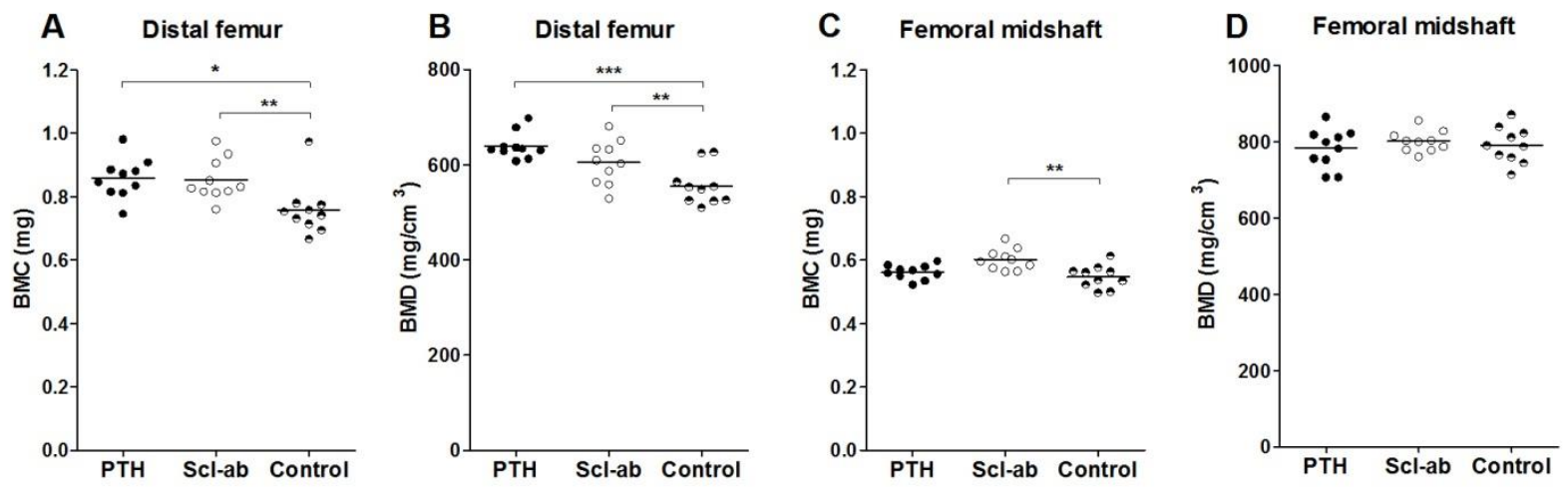

Figure 5: Bone mineral content (BMC) and bone mineral density (BMD) of the distal femur and femoral midshaft. A) Both PTH and Scl-ab significantly increased BMC in the distal femur compared to the control. B) In the distal femur both PTH and Scl-ab treatment increased BMD relative to control. C) In the femoral midshaft only Scl-ab had a significant effect on BMC compared to control. D) In the femoral midshaft no treatment had a significant effect on BMD. *, p < 0.05; **, p < 0.01; ***, p < 0.001 . 

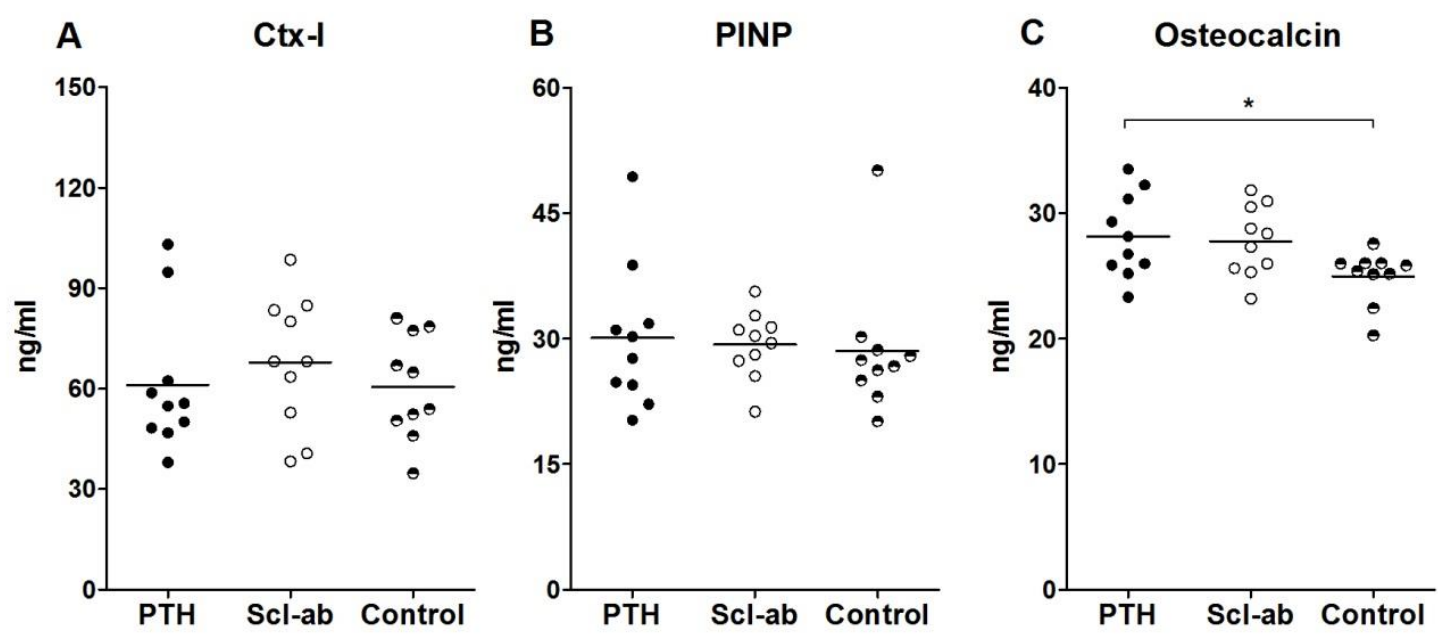

Figure 6: Serum biomarkers of bone turnover after 4 weeks of treatment. Both C-terminal telopeptide collagen (CTX-I) A) and procollagen type I N-terminal propeptide (PINP) B) appear unaffected by treatment. Only Osteocalcin C) levels were significantly elevated due to PTH treatment. *, p < 0.05; 


\section{Acknowledgement}

This study was funded by Lilly and the Swedish research council grant(VR 2009-6725). FA was funded by an award from Linkoping University and BM was funded by the National Science Foundation.

FA and BM declare no conflict of interest. MH, JL, MDA, KS, AH and MS are employees of Lilly. PA serves as a consultant and has received funding from Lilly. 


\section{References}

1. ten Dijke, P., et al., Osteocyte-derived sclerostin inhibits bone formation: its role in bone morphogenetic protein and Wnt signaling. J Bone Joint Surg Am, 2008. 90 Suppl 1: p. 31-5.

2. van Bezooijen, R.L., et al., SOST/sclerostin, an osteocyte-derived negative regulator of bone formation. Cytokine Growth Factor Rev, 2005. 16(3): p. 319-27.

3. Winkler, D.G., et al., Osteocyte control of bone formation via sclerostin, a novel BMP antagonist. Embo J, 2003. 22(23): p. 6267-76.

4. Chen, Y., et al., Beta-catenin signaling plays a disparate role in different phases of fracture repair: implications for therapy to improve bone healing. PLoS Med, 2007. 4(7): p. e249.

5. van Bezooijen, R.L., et al., Sclerostin in mineralized matrices and van Buchem disease. J Dent Res, 2009. 88(6): p. 569-74.

6. Beighton, P., L. Durr, and H. Hamersma, The clinical features of sclerosteosis. A review of the manifestations in twenty-five affected individuals. Ann Intern Med, 1976. 84(4): p. 393-7.

7. Hamersma, H., J. Gardner, and P. Beighton, The natural history of sclerosteosis. Clin Genet, 2003. 63(3): p. 192-7.

8. Balemans, W., et al., Identification of a $52 \mathrm{~kb}$ deletion downstream of the SOST gene in patients with van Buchem disease. J Med Genet, 2002. 39(2): p. 91-7.

9. Brunkow, M.E., et al., Bone dysplasia sclerosteosis results from loss of the SOST gene product, a novel cystine knot-containing protein. Am J Hum Genet, 2001. 68(3): p. 577-89.

10. Semenov, M., K. Tamai, and X. He, SOST is a ligand for LRP5/LRP6 and a Wnt signaling inhibitor. J Biol Chem, 2005. 280(29): p. 26770-5.

11. Agholme, F., et al., Sclerostin antibody treatment enhances metaphyseal bone healing in rats. J Bone Miner Res, 2010. 25(11): p. 2412-8.

12. Ominsky, M.S., et al., Inhibition of sclerostin by monoclonal antibody enhances bone healing and improves bone density and strength of non-fractured bones. $\mathrm{J}$ Bone Miner Res, 2011. 26(5): p.1012-21.

13. Andreassen, T.T., C. Ejersted, and H. Oxlund, Intermittent parathyroid hormone (1-34) treatment increases callus formation and mechanical strength of healing rat fractures. J Bone Miner Res, 1999. 14(6): p. 960-8.

14. Nakajima, A., et al., Mechanisms for the enhancement of fracture healing in rats treated with intermittent low-dose human parathyroid hormone (1-34). J Bone Miner Res, 2002. 17(11): p. 2038-47.

15. Skripitz, R., T.T. Andreassen, and P. Aspenberg, Strong effect of PTH (1-34) on regenerating bone: a time sequence study in rats. Acta Orthop Scand, 2000. 71(6): p. 619-24. 
16. Peichl, P., et al., Parathyroid hormone 1-84 accelerates fracture-healing in pubic bones of elderly osteoporotic women. J Bone Joint Surg Am, 2011. 93(17): p. 1583-7.

17. Skripitz, R. and P. Aspenberg, Early effect of parathyroid hormone (1-34) on implant fixation. Clin Orthop Relat Res, 2001(392): p. 427-32.

18. Skripitz, R. and P. Aspenberg, Implant fixation enhanced by intermittent treatment with parathyroid hormone. J Bone Joint Surg Br, 2001. 83(3): p. 437-40.

19. Aspenberg, P., et al., Teriparatide for acceleration of fracture repair in humans: a prospective, randomized, double-blind study of 102 postmenopausal women with distal radial fractures. J Bone Miner Res, 2010. 25(2): p. 404-14.

20. Aspenberg, P. and T. Johansson, Teriparatide improves early callus formation in distal radial fractures. Acta Orthop, 2010. 81(2): p. 234-6.

21. Sato, M., G.Q. Zeng, and C.H. Turner, Biosynthetic human parathyroid hormone (1-34) effects on bone quality in aged ovariectomized rats. Endocrinology, 1997. 138(10): p. 4330-7.

22. Agholme, F., et al., The effects of Dickkopf-1 antibody on metaphyseal bone and implant fixation under different loading conditions. Bone, 2011. 48(5): p. 988-96.

23. Wermelin, K., et al., Surface-bound bisphosphonates enhance screw fixation in rats--increasing effect up to 8 weeks after insertion. Acta Orthop, 2007. 78(3): p. 385-92.

24. Tashjian, A.H., Jr. and B.A. Chabner, Commentary on clinical safety of recombinant human parathyroid hormone 1-34 in the treatment of osteoporosis in men and postmenopausal women. J Bone Miner Res, 2002. 17(7): p. 1151-61.

25. Guo, J., et al., Suppression of Wnt signaling by Dkkl attenuates PTH-mediated stromal cell response and new bone formation. Cell Metab, 2010. 11(2): p. 161-71.

26. Bedi, B., et al., Silencing of parathyroid hormone (PTH) receptor 1 in T cells blunts the bone anabolic activity of PTH. Proc Natl Acad Sci U S A, 2012. 109(12): p. E725-33.

27. Kramer, I., et al., Parathyroid hormone (PTH)-induced bone gain is blunted in SOST overexpressing and deficient mice. J Bone Miner Res, 2010. 25(2): p. 17889.

28. Ominsky, M.S., et al., Inhibition of sclerostin by monoclonal antibody enhances bone healing and improves bone density and strength of nonfractured bones. $\mathrm{J}$ Bone Miner Res, 2011. 26(5): p. 1012-21.

29. Leupin, O., et al., Bone overgrowth-associated mutations in the LRP4 gene impair sclerostin facilitator function. J Biol Chem, 2011. 286(22): p. 19489-500.

30. Gardner, J.C., et al., Bone mineral density in sclerosteosis; affected individuals and gene carriers. J Clin Endocrinol Metab, 2005. 90(12): p. 6392-5.

31. van Lierop, A.H., et al., Patients with sclerosteosis and disease carriers: human models of the effect of sclerostin on bone turnover. J Bone Miner Res, 2011. 26(12): p. 2804-11. 
